\title{
Practical Bit Loading Schemes for Multi-Antenna Multi-User Wireless OFDM Systems
}

\author{
Diego Bartolomé ${ }^{1}$ and Ana I. Pérez-Neira ${ }^{2}$ \\ 1 Telecomm. Techno. Center of Catalonia (CTTC), c/ Gran Capita 2-4, 08034 Barcelona, Spain, dbartolome@ieee.org \\ 2 Technical University of Catalonia (UPC), c/ Jordi Girona 1-3, 08034 Barcelona, Spain, anuska@gps.tsc.upc.es \\ Tel: +34 932058564, Fax: +34 2058399
}

\begin{abstract}
This paper deals with practical multi-antenna multiuser OFDM systems. With the additional degrees of freedom of multiple antennas and multiple subcarriers, the performance might be enhanced, but the scheduling complexity might increase exponentially. Since the scheduling with realistic integer signal mappings is an NP-complete combinatorial problem, suboptimum solutions based on the scalar product are good candidates to yield a fast and realizable practical implementation. We propose afterwards a power reuse strategy to lowerthe computational complexity, and show that the amount of signaling can be reduced by forcing an equal mapping for all the users at the same subcarrier.
\end{abstract}

\section{INTRODUCTION}

Currently, wireless multi-carrier communications are widely deployed, and multiple antennas further enhance the system performance. With multiple users, the problem of allocating the users into subcarriers, antennas, and performing the convenient integer bit allocation is NP-complete [1]. We focus on the Access Point (AP) in the downlink of an OFDMbased Wireless LAN, such as IEEE 802.11a. Due to the NPcompleteness, the problem can be separated into two parts, namely the user grouping and the beamforming, power, and bit allocation for each of the groups at all the subcarriers.

In a realistic scenario, the AP shall distribute the $K$ users into groups of $Q$ for each subcarrier, note that $K>Q$. Since this problem is NP-complete [1], suboptimum solutions are adequate, see e.g. [2] for an example of the uplink of an SDMA/TDMA system. In [3] the authors extend [2] to take into account several QoS parameters. In this paper, we justify and propose a greedy algorithm based on the normalized scalar product to allocate the users into groups.

Then, for each group, it is meaningful to separate the transmit beamforming and the power (and bit) allocation [4]. A well-suited beamforming criterion is Zero Forcing (ZF) [5], which provides a reasonable performance loss with respect to optimum downlink beamforming e.g. [6] or dirty paper precoding [7]. On top of it, the AP shall perform the power (and bit) allocation. Without multiple antennas, several bit allocation strategies for multi-carrier systems have been developed since [8], see e.g. [9], [10], [11], or [12] and references therein. The use of a ZF transmit beamforming forces their modification to take into account the special characteristics

This work is partially supported by the CTTC, by the Spanish government under FIT-070000-2003-257 and TIC2002-04594-C02 (jointly financed by FEDER), and by the Catalan government thanks to grant 2003FI 00190. of the spatial diversity system. Therefore, we develop in this paper extensions of well-known algorithms for this multiantenna multi-user multi-carrier system. Furthermore, we describe simple power reuse schemes, and propose a solution to reduce the amount of signaling, which affects the fairness.

\section{Problem Statement}

Boldface capital (lowercase) letters refer to matrices (vectors). The conjugate transpose of $\mathbf{a}$ is $\mathbf{a}^{H}$ and the element at row $i$ and column $j$ of $\mathbf{A}$ is denoted by $[\mathbf{A}]_{i, j}$. The square matrix with the diagonal given by $a_{1}, a_{2}, \ldots, a_{n}$ is $\operatorname{diag}\left(a_{1}, a_{2}, \ldots, a_{n}\right)$. The cardinality of the set $\mathcal{K}$ is expressed as $|\mathcal{K}|$. $\operatorname{det}(\mathbf{A})$ is the determinant of $\mathbf{A}$, and $\operatorname{tr}(\mathbf{A})$ is the trace. The vector $\mathbf{1}_{k}$ has zeros at all positions but the $k$ th, and $\exp (x)$ is the exponential function of $x$. We assume $M$ available constellations, using a different number of bits per symbol contained in the ordered set $\mathcal{M}$.

The system is summarized in Fig. 1. The ultimate purpose of the scheduling is to distribute the $K$ users in the cell into groups at every subcarrier, so that they can be served simultaneously by the $Q$-antenna AP $(K>Q)$. Since multi-carrier modulations are well-known, the signal model in this section is devoted exclusively to the frequency domain representation. Moreover, since the optimization procedures are performed instantaneously, we omit the time index in (1). Assuming that the $N$ subcarriers have their particular set of users $\mathcal{K}_{n}$ to be served, the signal at subcarrier $n$ is given by

$$
\mathbf{y}\left(\mathcal{K}_{n}\right)=\mathbf{H}\left(\mathcal{K}_{n}\right) \mathbf{B}\left(\mathcal{K}_{n}\right) \mathbf{s}\left(\mathcal{K}_{n}\right)+\mathbf{w}\left(\mathcal{K}_{n}\right) \in \mathbb{C}^{\left|\mathcal{K}_{n}\right| \times 1},
$$

where $\mathcal{K}_{n}$ emphasizes that the signal model is expressed for subcarrier $n$ and for the simultaneously-served users in $\mathcal{K}_{n}$. The $k$ th position of $\mathbf{y}\left(\mathcal{K}_{n}\right)\left(\mathbf{s}\left(\mathcal{K}_{n}\right)\right)$ is the received (transmitted) signal for user $k$ in the set $\mathcal{K}_{n}$ at the subcarrier $n$. The transmit beamvectors are gathered in $\mathbf{B}\left(\mathcal{K}_{n}\right)=$ $\left[\mathbf{b}_{1}\left(\mathcal{K}_{n}\right) \mathbf{b}_{2}\left(\mathcal{K}_{n}\right) \ldots \mathbf{b}_{K}\left(\mathcal{K}_{n}\right)\right] \in \mathbb{C}^{Q \times K} . \mathbf{H}\left(\mathcal{K}_{n}\right)$ is the $\left|\mathcal{K}_{n}\right| \times$ $Q$ complex flat-fading channel matrix at the $n$th subcarrier, the $k$ th row of which contains the $1 \times Q$ vector of the channel gains for the $k$ th user at the $n$th subcarrier, i.e. $\mathbf{h}_{k, n}^{T}$, which is obtained evaluating the Fourier transform of the $L$-tap channel vector $\mathbf{h}_{k}^{t}$ at the $n$th subcarrier, i.e. $\mathbf{f}_{n}^{H} \mathbf{h}_{k}^{t}$, where $\mathbf{f}_{n}^{H}=$ $\left[\begin{array}{llll}1 & \exp (-j 2 \pi n / N) & \ldots & \exp (-j 2 \pi n(L-1) / N)\end{array}\right]$. We assume that the channels $\mathbf{h}_{k}^{t}$ are independent, and perfectly known at the AP. The noise $\left[\mathbf{w}\left(\mathcal{K}_{n}\right)\right]_{k}$ are independent zeromean complex Gaussian random variables with variance $\sigma_{k, n}^{2}$. 
As in other papers such as [5] or [13], we assume a ZF transmit beamforming, which is equivalent to the MMSE for a low number of users and also for high SNR [14]. Moreover, it provides a reasonable degradation with respect to the optimum sum capacity as shown in [15]. ZF implies that, for each subcarrier, the $\left|\mathcal{K}_{n}\right|$ users see parallel and orthogonal fading channels corrupted only by AWGN and not by interference signals from other users. The transmit beamvector is normalized, so that the gain of the equivalent spatial channel is captured by $\alpha_{k}\left(\mathcal{K}_{n}\right)$, and the ZF criterion is finally expressed as $\mathbf{H}\left(\mathcal{K}_{n}\right) \widetilde{\mathbf{b}}_{k}\left(\mathcal{K}_{n}\right)=\alpha_{k}\left(\mathcal{K}_{n}\right) \mathbf{1}_{k}$ for each subcarrier and for each user. The normalized beamvector is

$$
\widetilde{\mathbf{b}}_{k}\left(\mathcal{K}_{n}\right)=\alpha_{k}\left(\mathcal{K}_{n}\right) \mathbf{H}^{H}\left(\mathcal{K}_{n}\right)\left(\mathbf{H}\left(\mathcal{K}_{n}\right) \mathbf{H}^{H}\left(\mathcal{K}_{n}\right)\right)^{-1} \mathbf{1}_{k},
$$

where the $\alpha_{k}^{2}\left(\mathcal{K}_{n}\right)=1 /\left[\left(\mathbf{H}\left(\mathcal{K}_{n}\right) \mathbf{H}^{H}\left(\mathcal{K}_{n}\right)\right)^{-1}\right]_{k, k}$. The matrix $\widetilde{\mathbf{B}}\left(\mathcal{K}_{n}\right)=\left[\widetilde{\mathbf{b}}_{1}\left(\mathcal{K}_{n}\right) \widetilde{\mathbf{b}}_{2}\left(\mathcal{K}_{n}\right) \ldots \widetilde{\mathbf{b}}_{\left|\mathcal{K}_{n}\right|}\left(\mathcal{K}_{n}\right)\right]$ gathers the $\left|\mathcal{K}_{n}\right|$ normalized beamvectors, thus $\mathbf{H}\left(\mathcal{K}_{n}\right) \widetilde{\mathbf{B}}\left(\mathcal{K}_{n}\right)=$ $\boldsymbol{\Lambda}\left(\mathcal{K}_{n}\right)=\operatorname{diag}\left(\alpha_{1}\left(\mathcal{K}_{n}\right), \alpha_{2}\left(\mathcal{K}_{n}\right), \ldots, \alpha_{\left|\mathcal{K}_{n}\right|}\left(\mathcal{K}_{n}\right)\right)$. Since the beamforming matrix also contains the power allocation $\beta_{k}\left(\mathcal{K}_{n}\right)$, then $\mathbf{B}\left(\mathcal{K}_{n}\right)=\widetilde{\mathbf{B}}\left(\mathcal{K}_{n}\right) \boldsymbol{\Gamma}\left(\mathcal{K}_{n}\right)$, where $\boldsymbol{\Gamma}\left(\mathcal{K}_{n}\right)=$ $\operatorname{diag}\left(\beta_{1}\left(\mathcal{K}_{n}\right), \beta_{2}\left(\mathcal{K}_{n}\right), \ldots, \beta_{\left|\mathcal{K}_{n}\right|}\left(\mathcal{K}_{n}\right)\right)$. The signal model in (1) can be finally reduced to a very simple expression

$$
\mathbf{y}\left(\mathcal{K}_{n}\right)=\mathbf{\Lambda}\left(\mathcal{K}_{n}\right) \boldsymbol{\Gamma}\left(\mathcal{K}_{n}\right) \mathbf{s}\left(\mathcal{K}_{n}\right)+\mathbf{w}\left(\mathcal{K}_{n}\right),
$$

or $y_{k}\left(\mathcal{K}_{n}\right)=\alpha_{k}\left(\mathcal{K}_{n}\right) \beta_{k}\left(\mathcal{K}_{n}\right) s_{k}\left(\mathcal{K}_{n}\right)+w_{k}\left(\mathcal{K}_{n}\right), \forall k \in \mathcal{K}_{n}$, in which the $\alpha_{k}^{2}\left(\mathcal{K}_{n}\right)$ are independent but concentrate the effect of the choice of the $\left|\mathcal{K}_{n}\right|$ users that are simultaneously served at the $n$th subcarrier, because they depend on the inverse of the matrix $\mathbf{H}\left(\mathcal{K}_{n}\right) \mathbf{H}^{H}\left(\mathcal{K}_{n}\right)$. Indeed, if the rows of $\mathbf{H}\left(\mathcal{K}_{n}\right)$ are highly correlated, i.e. the channels refer to a close spatial zone, more power is needed. Assuming unitary mean energy symbols, particularly, normalized QAM. For simplicity and for a real-time implementation of an scheduler, we use the approximate BER for QAM signals [16]

$$
\operatorname{BER}_{k}\left(\mathcal{K}_{n}\right) \approx c_{1} \exp \left(-\frac{c_{2} \gamma_{k}\left(\mathcal{K}_{n}\right)}{2^{m_{k}\left(\mathcal{K}_{n}\right)}-1}\right),
$$

where $m_{k}\left(\mathcal{K}_{n}\right)$ is the number of bits per symbol of the mapping used at the $n$th subcarrier by user $k, c_{1}=0.2$, $c_{2}=1.6$, and $\gamma_{k}\left(\mathcal{K}_{n}\right)$ refers to the SNR for the $k$ th user at subcarrier $n$, i.e. $\gamma_{k}\left(\mathcal{K}_{n}\right)=\alpha_{k}^{2}\left(\mathcal{K}_{n}\right) \beta_{k}^{2}\left(\mathcal{K}_{n}\right) / \sigma_{k, n}^{2}\left(\mathcal{K}_{n}\right)$. In an optimistic situation, the number of bits per symbol might change at every subcarrier and for every user. Our general problem consists of maximizing the total discrete achievable rate for all the users at all the subcarriers, i.e.

$$
\begin{aligned}
\max & \sum_{n=0}^{N-1} \sum_{k \in \mathcal{K}_{n}} m_{k}\left(\mathcal{K}_{n}\right) \\
\text { s.t. } & \sum_{n=0}^{N-1} \sum_{k \in \mathcal{K}_{n}} \beta_{k}^{2}\left(\mathcal{K}_{n}\right) \leq P_{T}, 0 \leq n \leq N-1 \\
& \operatorname{BER}_{k}\left(\mathcal{K}_{n}\right) \leq \mathrm{BER}_{t}, 0 \leq n \leq N-1, \forall k \in \mathcal{K}_{n}, \\
& m_{k}\left(\mathcal{K}_{n}\right) \in \widetilde{\mathcal{M}}, 0 \leq n \leq N-1, \forall k \in \mathcal{K}_{n}
\end{aligned}
$$

where, due to algorithmic issues, the set $\widetilde{\mathcal{M}}$ is defined as the union of the possible constellations together with 0 (no

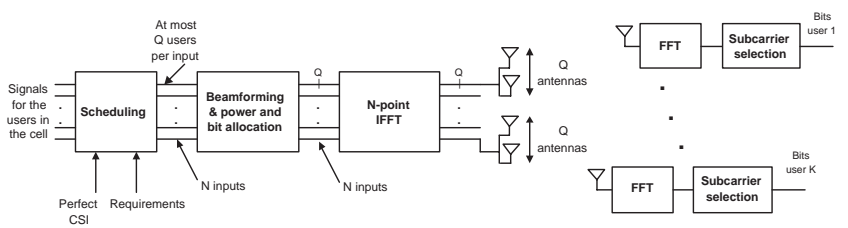

Fig. 1. Block diagram of the system: with perfect Channel State Information (CSI), the AP clusters the users into groups, i.e. the scheduling task. For each group at each of the $N$ subcarriers, the AP performs the $\mathrm{ZF}$ beamforming, and the power and bit allocation. The $N$ inputs of at most $Q$ users feed the IFFT block of OFDM. At the receivers, terminals demodulate only their signals. We assume that this information is sent by the AP through a broadcast channel.

transmission), that is, $\widetilde{\mathcal{M}}=\{0\} \cup \mathcal{M}$. The user and subcarrier BER constraint in (7) allows to find a direct expression for the power by substituting (4) into (7), i.e.

$$
\beta_{k, n}^{2}=\frac{\sigma_{k, n}^{2}\left(2^{m_{k}\left(\mathcal{K}_{n}\right)}-1\right)}{c_{2} \alpha_{k}^{2}\left(\mathcal{K}_{n}\right)} \log \left(\frac{c_{1}}{\mathrm{BER}_{t}}\right),
$$

which can be inserted into (6) to compute the total used power. The objective in (5)-(8) involves mainly two tasks: i) the grouping of the users in the sets $\mathcal{K}_{n}$ at the $N$ subcarriers, and ii) the space-frequency bit allocation. In the remainder of this section, we justify the use of the scalar product to allocate the users at the subcarriers.

\section{A. Towards a simple tool to the user clustering}

As stated, the objective in (5)-(8) is NP-complete [1], which means that it cannot be solved in polynomial time, i.e. the complexity increases exponentially with the number of variables. It is shown in [2] by graph theory that the problem of minimizing the length of an SDMA/TDMA frame, while ensuring a minimum SINR for each terminal, is NP-complete. In our case, the proof follows directly from linear programming, in which several combinatorial problems are known to be NP-complete, among others the Knapsack. Without going into the details, the maximization in (5)-(8) can be mapped into the well-known Knapsack problem, thus it is NP-complete.

To build real-time channel-adapted realizable schedulers, suboptimum algorithms are therefore motivated. Several papers have proposed the scalar product of the channel vectors among the users as a well-suited approach for the design of schedulers, see e.g. [4]. We prove next that this approach is a valid path to separate the users intelligently. A similar concept of user separability is developed e.g. in [17], where heuristic algorithms are developed to study the impact of smart antennas on the Medium Access Control (MAC) layer, with especial emphasis on the limited availability of resources, i.e. they assume that only a finite set of beamvectors is available. A related concept based exclusively on power capture threshold is [18] for a sectorized AP. However, to the best of our knowledge, the normalized scalar product has not been yet shown to be a suited technique to separate the users.

To show the previous statement, we concentrate on a single subcarrier and omit the index $\mathcal{K}_{n}$ for simplicity. $K=2$ users are considered, denoted by the index $k=\{1,2\}$. Their channels are expressed as $\mathbf{h}_{k}$, and are gathered in the matrix $\mathbf{H}$. If the two users use the same signal mapping, it follows from (9) that $\sum \beta_{k}^{2} \propto \sum 1 / \alpha_{k}^{2}=\operatorname{tr}\left(\mathbf{H} \mathbf{H}^{H}\right)^{-1}$. For the proof, we shall first compute the determinant of the matrix 
$\mathbf{H} \mathbf{H}^{H}$, where $\mathbf{H}=\left[\mathbf{h}_{1} \mathbf{h}_{2}\right]^{T}$. After some manipulations, it yields $\operatorname{det}\left(\mathbf{H} \mathbf{H}^{H}\right)=\left\|\mathbf{h}_{1}\right\|^{2}\left\|\mathbf{h}_{2}\right\|^{2}-\left|\mathbf{h}_{1}^{H} \mathbf{h}_{2}\right|^{2}$. As stated, the consumed power is determined by the trace of the matrix $\left(\mathbf{H H}^{H}\right)^{-1}$, so we find

$$
\operatorname{tr}\left(\mathbf{H} \mathbf{H}^{H}\right)^{-1}=\frac{\left\|\mathbf{h}_{1}\right\|^{2}+\left\|\mathbf{h}_{2}\right\|^{2}}{\operatorname{det}\left(\mathbf{H} \mathbf{H}^{H}\right)}=\frac{\left\|\mathbf{h}_{1}\right\|^{-2}+\left\|\mathbf{h}_{2}\right\|^{-2}}{1-c_{1,2}^{2}},
$$

where $c_{1,2}=\frac{\left|\mathbf{h}_{1}^{H} \mathbf{h}_{2}\right|}{\left\|\mathbf{h}_{1}\right\|\left\|\mathbf{h}_{2}\right\|}$, and the second equality is obtained by dividing both the numerator and the denominator by $\left(\left\|\mathbf{h}_{1}\right\|\left\|\mathbf{h}_{2}\right\|\right)^{2}$. If there were $Q=2$ antennas, $c_{1,2}$ would reflect the cosinus of the angle between $\mathbf{h}_{1}$ and $\mathbf{h}_{2}$. Anyway, $c_{1,2}^{2}$ is the square of the normalized scalar product between $\mathbf{h}_{1}$ and $\mathbf{h}_{2}$ defined accordingly, and its range is $0 \leq c_{1,2} \leq 1$. The lower bound occurs if $\mathbf{h}_{1}$ and $\mathbf{h}_{2}$ are orthogonal, and the upper bound when $\mathbf{h}_{1}=\mathbf{h}_{2}$. With all this,

$$
\lim _{\left\|\mathbf{h}_{1}\right\| \rightarrow \infty} \operatorname{tr}\left(\mathbf{H} \mathbf{H}^{H}\right)^{-1}=\frac{\left\|\mathbf{h}_{2}\right\|^{-2}}{1-c_{1,2}^{2}}
$$

and it is easy to see that it is bounded if $\mathbf{h}_{1} \neq \mathbf{h}_{2}$. The same situation occurs if the limit is calculated for $\left\|\mathbf{h}_{1}\right\| \rightarrow$ $\infty$. However, if we compute the limit when $\mathbf{h}_{1} \rightarrow \mathbf{h}_{2}$, or equivalently when $c_{1,2} \rightarrow 1$, it yields

$$
\lim _{c_{1,2} \rightarrow 1} \operatorname{tr}\left(\mathbf{H} \mathbf{H}^{H}\right)^{-1}=\infty \text {. }
$$

Therefore, it is more critical to separate those users coming from the same zone of space rather than using the norm of their channel vector as a performance measure to allocate users. This justifies the use of a measure like $c_{1,2}$ as a way to allocate the users into the subcarriers. If more users form the matrix $\mathbf{H}$, the cost is determined by the one with a highest $c_{i, j}$.

\section{SPACE-Frequency Multi-USER SCHEDUling}

With the previous results, we propose a suboptimum yet very simple real-time approach based on the normalized scalar product to allocate the users into subcarriers in groups of $Q$, and then we deal with the spatial power and bit allocation, which is an extension to OFDM of the Maximum Sum Rate spatial bit allocation for single carrier developed in [19]. Finally, we propose practical schemes for comparison.

\section{A. User-subcarrier assignment based on the scalar product}

The initialization is the following: the AP computes the cost for each pair of users, $i$ and $j$, for all the subcarriers, thus $\mathcal{K}_{n}=\{i, j\}, \forall n$. However, since the channel is generally frequency-selective, the separation of the users depends on the particular subcarrier. Therefore, the associated cost of putting user $i$ and user $j$ together is determined by the maximum cost among the subcarriers. Indeed, this is the worst case among user $i$ and $j$, which is the limiting factor. Then,

$$
c_{i, j}=\max _{n} \frac{\left|\mathbf{h}_{i, n}^{H} \mathbf{h}_{j, n}\right|}{\left\|\mathbf{h}_{i, n}\right\|\left\|\mathbf{h}_{j, n}\right\|}, \forall i \neq j
$$

are computed for all pairs of users. The AP sorts then these values in descending order, and starts assigning the users with higher costs at adjacent subcarriers, until no carriers are left. After this procedure, each carrier is filled with a single user,
TABLE I

USER Clustering BASEd on SCALAR Product

1. Set $n=0$. The users for subcarrier $n$ are collected in $\mathcal{K}_{n}$, which has been initialized.

2. If $n=N-1$, finish. Otherwise, do $n \leftarrow n+1$.

3. Select $k: \min _{k} \max _{k^{\prime} \in \mathcal{K}_{n}} c_{k, k^{\prime}}$. Add user $k$ to $\mathcal{K}_{n}$, i.e. $\mathcal{K}_{n} \leftarrow \mathcal{K}_{n}+k$.

4. If $\left|\mathcal{K}_{n}\right|<Q$, go to step 3. Otherwise, go to step 2 .

and the AP shall fill the subcarriers until there are $Q$ users preallocated per subcarrier. One could use rather cost-extensive approaches such as those developed in [5], but the scalar product is a good and very simple option. The procedure is summarized in Table I, and it is very intuitive. When the AP has $Q$ pre-allocated users per subcarrier, it applies the MMSR.

\section{B. Multi-antenna Multi-carrier Maximum Sum Rate (MMSR)}

The space-frequency bit allocation has some differences with respect to traditional multi-carrier bit loading, e.g. the channels change accordingly when the users that are simultaneously served change, see also [19]. In realistic scenarios with several users, if the problem is not feasible, then the AP has to perform also the admission control, i.e. choose the users that will be served. Essentially, two strategies can be found in the single antenna bit loading literature in Section I, namely bit filling and bit removal. The former adds a bit to the user/subcarrier providing the lowest increase in total power, and bit removal schemes remove the most penalizing bit until the power constraint is fulfilled. With multiple antennas, it is not possible to do strict bit filling algorithms, since the interactions among the users that are being simultaneously served are crucial. For instance, the user with best channel might not even have a good channel when grouped together in an SDMA scheme [19].

The number of bits for user $k$ at subcarrier $n$ is $m_{k}\left(\mathcal{K}_{n}\right)$, except for the $l$ th, which changes the number to $m_{l}^{j}\left(\mathcal{K}_{n}\right)$ instead of $m_{l}^{i}\left(\mathcal{K}_{n}\right)$, where $m_{l}^{i}\left(\mathcal{K}_{n}\right)>$ $m_{l}^{j}\left(\mathcal{K}_{n}\right)$. The power saving $p_{l, n}\left(m_{l}^{i}\left(\mathcal{K}_{n}\right), m_{l}^{j}\left(\mathcal{K}_{n}\right)\right)$ can be approximated as $\sigma_{l, n}^{2} 2^{m_{l}^{i}\left(\mathcal{K}_{n}\right)}-2^{m_{l}^{j}\left(\mathcal{K}_{n}\right)} / \alpha_{k}^{2}\left(\mathcal{K}_{n}\right)$ if $m_{l}^{j}\left(\mathcal{K}_{n}\right) \in \mathcal{M}$, and as $\sum_{k \in \mathcal{K}_{n}} \sigma_{k, n}^{2} 2^{m_{k}\left(\mathcal{K}_{n}\right)} / \alpha_{k}^{2}\left(\mathcal{K}_{n}\right)-$ $\sum_{k \in \widetilde{\mathcal{K}}_{n}} \sigma_{k, n}^{2} 2^{m_{k}\left(\widetilde{\mathcal{K}}_{n}\right)} / \widetilde{\alpha}_{k}^{2}\left(\widetilde{\mathcal{K}}_{n}\right)$ if $m_{l}^{j}\left(\mathcal{K}_{n}\right) \notin \mathcal{M} . \widetilde{\mathcal{K}}_{n}$ gathers all the users but the $l$ th and the equivalent channels $\widetilde{\alpha}_{k}$ refer to the users in $\widetilde{\mathcal{K}}_{n}$. In fact, this is not the exact saving that is obtained because if a user is removed, then the rest have the chance to increase their modulation index. However, the complexity reduction of this approximation justifies its use.

The MMSR in Table II is based on a bit removal technique, but it is aided by a bit filling scheme, performed when the spatial channel gains $\alpha_{k}$ change, i.e. whenever the set of active users varies. Briefly, the MMSR first tries to serve all the users in the set $\mathcal{K}_{n}, \forall n$ obtained by the user-subcarrier clustering method with the highest modulation in $\mathcal{M}$ at steps $2-4$. If the power constraint in (6) is not fulfilled (step 5), the scheduler decides which user among all the carriers should reduce the constellation size or which user should not be served. Since the number of bits shall be reduced, the scheduler selects the user having a maximum incremental cost of using a lower modulation, i.e. the user that saves more power if the bit rate is 
TABLE II

SPACE-FrEQUENCY BIT ALlocAtion: MMSR AND MMMR

1. The set $\mathcal{K}_{n}$ is obtained by the user-subcarrier assignment.

2. Set $m_{k}\left(\mathcal{K}_{n}\right)=\max \mathcal{M}, \forall k \in \mathcal{K}_{n}, 0 \leq n \leq N-1$.

3. Build $\mathbf{H}\left(\mathcal{K}_{n}\right)$ and compute $\alpha_{k}^{2}\left(\mathcal{K}_{n}\right)$.

4. Compute $\beta_{k}^{2}\left(\mathcal{K}_{n}\right)$ according to (9), and the total used power $P_{S}=\sum_{n=0}^{N-1} \sum_{k \in \mathcal{K}_{n}} \beta_{k}^{2}\left(\mathcal{K}_{n}\right)$.

5. If $P_{S} \leq P_{T}$ or $\mathcal{K}_{n}=\emptyset, 0 \leq n \leq N-1$, then finish.

6. Compute $p_{k, n}\left(m_{k}^{i}\left(\mathcal{K}_{n}\right), m_{k}^{j}\left(\mathcal{K}_{n}\right)\right), 0 \leq n \leq N-1, \forall k \in$ $\mathcal{K}_{n}$, where $m_{l}^{i}\left(\mathcal{K}_{n}\right)$ is the current mapping and $m_{l}^{j}\left(\mathcal{K}_{n}\right)$ the lower one in $\mathcal{M}$.

7. Select $\{n, k\}: \max _{n} \max _{k} p_{k, n}\left(m_{k}^{i}\left(\mathcal{K}_{n}\right), m_{k}^{j}\left(\mathcal{K}_{n}\right)\right)$, and reduce the number of bits $m_{k}^{i}\left(\mathcal{K}_{n}\right) \leftarrow m_{k}^{j}\left(\mathcal{K}_{n}\right)$.

8. Only for MMMR: $m_{k}^{i}\left(\mathcal{K}_{n}\right) \leftarrow m_{k}^{j}\left(\mathcal{K}_{n}\right), \forall k \in \mathcal{K}_{n}$.

9. If $m_{k}^{i}\left(\mathcal{K}_{n}\right) \in \mathcal{M}$, go to step 4. Else, $\mathcal{K}_{n} \leftarrow \mathcal{K}_{n}-k$, set $m_{k}\left(\mathcal{K}_{n}\right)=\max \mathcal{M}, \forall k \in \mathcal{K}_{n}$, and go to step 3 .

reduced, see steps 6 and 7. The AP reduces the number of bits of the selected user, and if it belongs to a possible constellation the algorithm goes again to step 4. Otherwise, it drops that user out from the set of active users at the corresponding subcarrier, and the constellation size of all the remaining users in that subcarrier is set again to the maximum (step 9). The algorithm finishes when the power constraint is fulfilled or when the set of active users for all the subcarriers is empty (step 5). In that case, that subcarrier remains unused.

\section{Power Reuse}

The complexity of (5)-(8) might be reduced by separating the problem into smaller ones, i.e. solve the problem independently at each subcarrier. With a low $K$, we could even perform the Exhaustive Search (ES) for each subcarrier. Since a significant amount of power might remain unused, some kind of power reuse scheme is needed. The procedure is very simple: we perform the ES at each subcarrier with an available power of $P_{T} / N+P_{n}$, where $P_{n}$ gathers the accumulated unused power for the subcarriers previous to the $n$ th. Then, an extremely simple scheme is obtained, when compared to the iterative (and far more complex) power redistribution routine for the eigenmodes of single link MIMO system in [20].

The same idea is valid for the previous MMSR performed independently for each subcarrier. The simplest scheme is the Random Approach, which selects a random combination of the set of active users and then performs with them the proposed MMSR spatial bit allocation for each subcarrier with the power reuse. We shall also consider Opportunistic communications. With multiple antennas, we consider that it refers to the fact that the spatial diversity is used to enhance the SNR at the receiver, but there is no (user) multiplexing gain. Only the user with the best norm of the channel, that with $\max _{k}\left\|\mathbf{h}_{k}\right\|$, is scheduled for transmission, with the highest number of bits per symbol satisfying the BER constraint.

\section{Reducing the signaling needs}

The amount of overhead required might have relevance especially when the number of transmitted OFDM symbols is low, and might deeply penalize the performance. If $b$ bits are required to transmit the desired constellation to the users, a total number of $b_{T}=Q \times N \times b$ bits are needed for signaling at every burst. Among other simple practical options to reduce the signaling needs, the AP might force an equal signal mapping for all the users at the same subcarrier, i.e. the Multicarrier Maximization of the Minimum Rate (MMMR). This might reduce the overhead by a maximum factor of $Q$ (upper bound). In fact, by using the same mapping for all the users we guarantee that the users being served receive the same rate. This ensures the fairness among users if they are homogeneous (or pay the same price for the service). In some sense, we maximize the number of users that are served but the global performance might be penalized. Fairness issues have been rarely evaluated in the literature, see e.g. [21], or the simplified approach in [14]. A theoretical study of fairness in multiantenna multi-user channels is conducted in [22]. The MMMR alternative can be expressed as a max-min problem, i.e.

$$
\begin{aligned}
& \max _{m_{k}\left(\mathcal{K}_{n}\right)} \min _{k} m_{k}\left(\mathcal{K}_{n}\right), 0 \leq n \leq N-1, \\
& \text { s.t. }(6),(7) \text {, and (8). }
\end{aligned}
$$

Again, the optimum solution for this problem implies the exhaustive search among all the users and all the number of bits. The Multi-carrier Maximization of the Minimum Rate (MMMR) algorithm is summarized in Table II, which consists essentially of the same steps as the MMSR, but in this case, all the users at subcarrier $n$ lower their modulation size when the selected user is at the same subcarrier, see step 8 .

\section{Performance Evaluation}

Simulation are conducted for a typical office environment with $50 \mathrm{~ns}$ average rms delay spread for OFDM-based Wireless LAN, model A in [23]. We assume for simulation purposes that the noise is equal for all subcarriers $\sigma_{k, n}^{2}=$ $\sigma^{2}, \forall n, \forall k$. We define the SNR as the ratio $P_{T} / \sigma^{2}$. We assume $N=64$ subcarriers, and QAM constellations with $\mathcal{M}=$ $\{2,4,6\}$ bits per symbol. The BER target is $1 e-4$.

First, we compare the MMSR to the strategies with a simple power reuse. We plot in Fig. 2 the average throughput at the physical layer in terms of number of bits per symbol per subcarrier vs. the SNR, when $K=5$ and $Q=3$. We wee that the Exhaustive Search (ES) scheme, with or without power reuse, outperforms the other methods in the high signal to noise ratio range at the expense of a prohibitive computational complexity when $K$ is high. The performance of the MMSR has practically no degradation at low SNR, but differences are higher when the SNR increases. The simple scheme with power reuse is very close to the globally computed maximum sum rate. If one performed the schemes individually per subcarrier and without power reuse, some power would be wasted, thus the rate would be penalized. The suboptimum user-subcarrier clustering based on the scalar product provides a reasonable trade-off between performance and complexity.

Second, we simulate a more realistic scenario with $K=20$ users and the same number of antennas. We plot in Fig. 3 the average throughput at the physical layer in terms of number of bits per symbol per subcarrier vs. the SNR. We see again that the performance with the globally computed algorithms is very close to the use of the simple power reuse. If we impose equal constellation for all users at a certain subcarrier, i.e. the MMMR, the performance is penalized with respect to the 
MMSR, but the amount of signaling is also reduced and the AP guarantees fairness. In any case, a noticeable gain is achieved with respect to a random selection of users. Finally, one can see that opportunistic communications yields a low throughput at high SNR because of the limitation of the scheduling of a single user. However, at low SNR where the noise is dominates the performance tends to be optimum, see [7] or [24]. In that region, typically a single user is scheduled per subcarrier at most, therefore the MMMR is equivalent to the MMSR.

\section{CONCLUSIONS}

We have studied the practical implementation of bit allocation techniques with a combination of space and frequency diversity. We have reviewed the particular issues that arise with the spatial dimension, and have shown that the objective is NP-complete. After proving that solutions based on the scalar product are good candidates for a fast and realizable crosslayer scheduler, we have described a very simple strategy. We have proposed a mechanism to reduce the huge signaling needs of the multi-user multi-carrier spatial bit allocation, which has also fairness implications. By means of realistic simulations for typical indoor Wireless LAN environments, we have shown that simple practical schemes might be adequate in the design of schedulers. Besides the trade-off between performance and complexity, there exists the trade-off between performance and signaling, and between global and the individual needs. For this reasons, the final choice strongly depends on the AP.

\section{REFERENCES}

[1] M. Garey and D. Johnson, Computers and intractability: A guide to the theory of NP-completeness. Freeman, 1979.

[2] F. Shad, T.D. Todd, V. Kezys, and J. Litva, "Dynamic Slot Allocation (DSA) in Indoor SDMA/TDMA Using a Smart Antenna Basestation," IEEE/ACM Trans. Networking, vol. 9, no. 1, Feb. 2001.

[3] H. Yin and H. Liu, "Performance of Space-Division Multiple-Access (SDMA) with Scheduling," IEEE Trans. Wireless Commun., vol. 1, no. 4, pp. $611-618$, Oct. 2002.

[4] D. Bartolome, D.P. Palomar, A.I. Perez-Neira, "Real-Time Scheduling for Wireless Multiuser MISO Systems under Different Fairness Criteria," in Proc. Intern. Symp. on Signal Proc. and its Appl. (ISSPA), Jul. 2003.

[5] D. Bartolome, A. Pascual Iserte, A.I. Perez-Neira, "Spatial Scheduling Algorithms for Wireless Systems," in Proc. of International Conference on Acoustics, Speech, and Signal Proc. (ICASSP), Apr. 2003.

[6] F. Rashid-Farrokhi, K.J. Ray Liu, and L. Tassiulas, "Transmit Beamforming and Power Control for Cellular Wireless Systems," IEEE J. Select. Areas Commun., vol. 16, no. 8, pp. 1437-1449, October 1998.

[7] G. Caire and S. Shamai, "On the achievable Throughput of a Multiantenna Gaussian Broadcast Channel," IEEE Trans. Inform. Theory, vol. 49, no. 7, pp. 1691 - 1706, July 2003.

[8] D. Hughes-Hartogs, "Ensemble modem structure for imperfect transmission media," U.S. Patents 4679227 (07-1987) and 4731816 (03-1988).

[9] M. Zwingelstein-Colin, M. Gazalet, and M. Gharbi, "Non-iterative bit-loading algorithm for ADSL-type DMT applications," IEE Proc. Commun., vol. 150, no. 6, Dec. 2003.

[10] D. Kivanc, G. Li, and H. Liu, "Computationally Efficient Bandwidth Allocation and Power Control for OFDMA," IEEE Trans. Wireless Commun., vol. 2, no. 6, Nov. 2003.

[11] C.Y. Wong, R.S. Cheng, K.B. Letaief, and R.D. Murch, "Multiuser OFDM with Adaptive Subcarrier, Bit, and Power Allocation," IEEE J. Select. Areas Commun., vol. 17, no. 10, Oct. 1999.

[12] J. Lee, R. V. Sonalkar, and J. M. Cioffi, "Multi-user bit-loading for multi-carrier systems," submitted to IEEE Trans. Commun., 2003.

[13] D. Bartolome and A.I. Perez-Neira, "Performance Analysis of Scheduling and Admission control for Multiuser Downlink SDMA," in Proc. of Int. Conf. on Acoustics, Speech, and Signal Proc. (ICASSP), May. 2004.

[14] S. Thoen, L. Van der Perre, M. Engels, and H. De Man, "Adaptive Loading for OFDM/SDMA-Based Wireless Networks," IEEE Trans. Commun., vol. 50, no. 11, Nov. 2002.

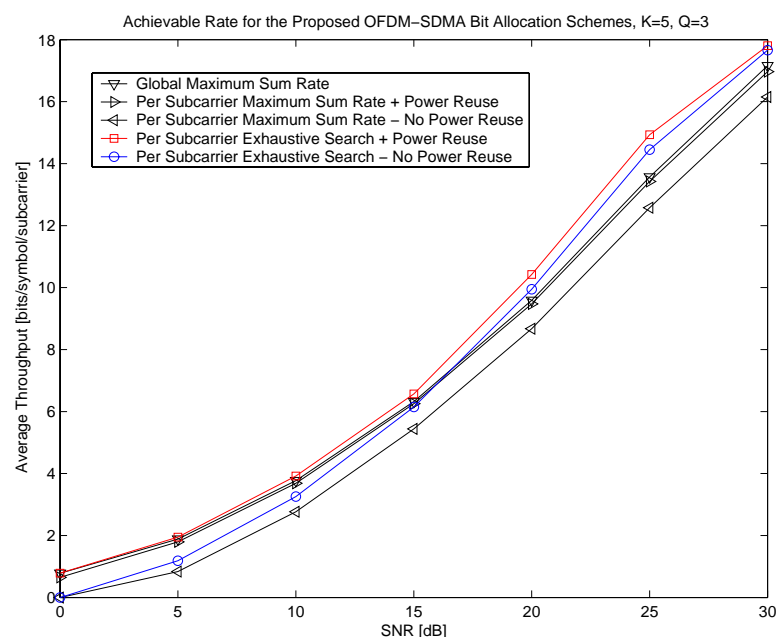

Fig. 2. Comparison in terms of throughput vs. the ratio $S N R=P_{T} / \sigma^{2}$ to evaluate the power reuse, with $K=5$ and $Q=3$.

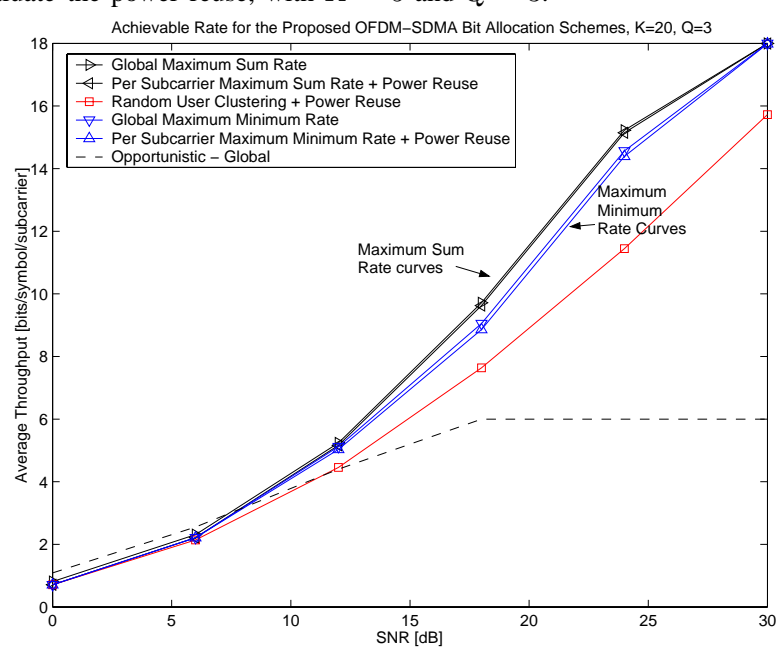

Fig. 3. Comparison in terms of throughput vs. the ratio $S N R=P_{T} / \sigma^{2}$ in a more realistic case, $K=20$ and $Q=3$.

[15] G. Dimic and N.D. Sidiropoulos, "Low-complexity downlink beamforming for maximum sum capacity," in Proc. of International Conference on Acoustics, Speech, and Signal Processing (ICASSP), May 2004.

[16] S.T. Chung and A.J. Goldsmith, "Degrees of Freedom in Adaptive Modulation: A Unified View," IEEE Trans. Commun., vol. 49, no. 9, pp. 1561-1571, Sept. 2001.

[17] I. Koutsopoulos, T. Ren, and L. Tassiulas, "Adaptive Channel Allocation for OFDM-based Smart Antenna Systems with Limited Transceiver Resources," Tech. Rep., Univ. of Maryland, 2002.

[18] A.S. Macedo and E.S. Sousa, "Antenna-Sector Time-Division Multiple Access for Broadband Indoor Wireless Systems," IEEE J. Select. Areas Commun., vol. 16, no. 6, Aug. 1998.

[19] D. Bartolome and A. I. Perez-Neira, "Multiuser Spatial Scheduling in the Downlink of Wireless Systems," IEEE Sensor Array and Multichannel Signal Processing Workshop (SAM), Jul. 2004.

[20] X. Zhang and B. Ottersten, "Power Allocation and Bit Loading for Spatial Multiplexing in MIMO Systems," in Proc. of International Conference on Acoustics, Speech, and Signal Proc. (ICASSP), 2003.

[21] M. Ergen, S. Coleri, and P. Varaiya, "QoS Aware Adaptive Resource Allocation Techniques for Fair Scheduling in OFDMA Based Broadband Wireless Access Systems," IEEE Trans. Broadcast., Dec. 2003.

[22] D. Bartolome ans A.I. Perez-Neira," "Cross-Layer Design in MultiAntenna Multi-User Channels: A Unified Framework for Fairness," submitted to IEEE Trans. Wireless Commun., Apr. 2004.

[23] J. Medbo and P. Schramm, "Channel Models for Hiperlan/2 in Different Indoor Scenarios," BRAN 3ERIO85B, Mar. 1998.

[24] R. Knopp and P.A. Humblet, "Information capacity and power control in single-cell multiuser communications," in Proc. of IEEE Int. Conf. on Commun. (ICC), Jun. 1995. 